\title{
MRI and X-ray in axial spondyloarthritis: the relationship between inflammatory and structural changes
}

Walter P Maksymowych*

\begin{abstract}
Demonstration of an association between inflammation and spinal ankylosis has been challenging. Until the advent of MRI, prospective study was not possible due to inaccessibility of tissue. Recent studies using MRI have described an association between the presence of bone edema at vertebral corners on MRI and the subsequent development of syndesmophytes at the corresponding vertebral corners on radiography. Although reports have also highlighted the development of new syndesmophytes where the baseline MRI shows no inflammation, MRI has limited sensitivity for detection of spinal inflammation that is clearly evident on histopathology. There are also crucial methodological challenges because radiographic assessment is limited to the anterior corners of the cervical and lumbar spine while MRI lesions in the cervical spine are often small while spurious inflammatory signal is common in the lumbar spine. Follow-up MRI evaluation in two independent studies has also shown that inflammatory lesions that resolve after anti-TNF therapy are more prone to develop into syndesmophytes. It may be possible that very early inflammatory lesions resolve completely without sequelae if anti-TNF therapy is introduced before new bone formation becomes largely autonomous. For an individual patient the overall development of new bone during anti-TNF therapy may therefore depend on the balance between the number of early and more mature inflammatory lesions. Clinical trials of anti-TNF agents in early spondyloarthritis together with prospective MRI studies will allow more detailed testing of this hypothesis as a major priority for the research agenda in spondyloarthritis.
\end{abstract}

*Correspondence: walter.maksymowych@ualberta.ca 562 Heritage Medical Research Building, University of Alberta, Edmonton, Alberta, T6G 2S2 Canada

\section{Introduction}

A hallmark pathological feature of spondyloarthritis (SpA) is the development of ankylosis in axial joints. This process is the primary reason for the development of major disability. Its prevention therefore constitutes a key goal of management. There has been a long-standing assumption that inflammation is the process that sets in motion the chain of events that leads to ankylosis and that the two processes continue to be inextricably linked as the disease progresses over time. However, direct testing of this hypothesis has been difficult due to several factors, such as the lack of availability of objective clinical and laboratory measures of inflammation, delay in diagnosis, the slow progression of disease, and the lack of access to tissue for direct histopathological analysis. Investigators have turned to indirect approaches such as animal models of SpA and the testing of various biomarkers reflecting inflammation. The significant limitations of these approaches are not the subject of this review but highlight the increasing importance of recent advances in non-invasive assessment with imaging modalities. In particular, the advent of fat-suppressed magnetic resonance imaging (MRI) allows direct visualization of features related to inflammation and this has, for the first time, allowed the prospective non-invasive evaluation of inflammation in axial joints. The reports of these studies assessing the relationship between inflammation and structural changes have generated considerable interest, culminating in numerous symposia at international meetings. This review sets out the reasons why this topic has generated so much interest, outlines the methodology and pitfalls surrounding the use of MRI for evaluation of the axial joints, reviews the prospective studies that focused on MRI inflammation and its relation to radiographic changes, and suggests possible avenues of further research in SpA.

\section{Evidence for 'uncoupling' between inflammation and structural damage in spondyloarthritis}

The detailed histopathological examination of necropsy material and tissue biopsies outlined by John Ball in his 
1970 Heberden oration lent strong support to the concept that ankylosis was an excessive reparative response to an inflammatory and osteo-destructive process [1]. It was expected, therefore, that anti-TNF $\alpha$ therapies would be beneficial in preventing ankylosis in SpA through their profound ability to suppress inflammation. However, the results of three studies that compared radiographic progression over the minimum required 2 year time frame in patients in anti-TNF $\alpha$ trials with a historical cohort of ankylosing spondylitis (AS) patients did not support a beneficial effect of anti-TNF $\alpha$ therapy [2-4]. Several important limitations included the lack of comparability in disease severity, inadequate sample size, insufficient suppression of inflammation, and possible differences in intake of non-steroidal anti-inflammatory agents (NSAIDs), which have been shown to ameliorate progression [5]. Nevertheless, various sensitivity analyses aimed at demonstrating differences in progression within subgroups failed to identify any impact of anti-TNF $\alpha$ therapy. Assessment of anti-TNF $\alpha$ therapy in an animal model of SpA, ankylosing enthesitis, also failed to demonstrate amelioration of ankylosis by anti-TNF $\alpha$ therapy [6]. This led to an alternative hypothesis whereby an as yet unknown pathogenic trigger(s) simultaneously induces both an acute inflammatory reaction and activation of stromal progenitor cells, which ultimately leads to endochondral new bone [7]. But the processes of inflammation and new bone formation proceed along essentially disconnected or 'uncoupled' pathways once each has been activated.

Discerning the true relationship between inflammation and ankylosis is pivotal to future advances in disease modification because if these processes are largely uncoupled, future therapies will need to target bone formation pathways. A major challenge in understanding the link between these two processes in human SpA is the delayed recognition of disease so that most patients are assessed when both inflammatory and reparative processes are well established. It has been proposed that initial development of inflammation leads to erosive cartilage bone destruction followed by tissue repair and ultimately ossification once inflammation has completely resolved [8]. Consequently, this hypothesis would imply that early and effective suppression of inflammation would prevent the development of ankylosis. MRI allows non-invasive assessment of inflammation but the optimal approach to the assessment of bone is still radiography. Consequently, recent prospective studies have combined assessment of the evolution of inflammatory lesions using MRI with the development of new bone using radiography in the spine and sacroiliac joints (SIJs) to provide further insights regarding these hypotheses.

\section{MRI evaluation: standardization of methodology and key pitfalls}

As the diversity of acute and structural lesions on MRI at different stages of disease has become evident, there has been recognition for the need to develop a standardized methodology for assessment of MRI scans in SpA and to develop and validate standardized definitions in order to facilitate clinical research. This need is particularly relevant for assessment of the SIJs due to the complex anatomy of the joint. An international working group from Canada, Denmark, and Switzerland (The MORPHO Group) has developed a standardized module (available online at [9]) for evaluation of the SIJ [10]. The SIJs are scanned in the tilted semi-coronal plane and assessment of consecutive slices is undertaken from anterior to posterior according to anatomical landmarks and specific rules [11]. It is deemed essential that assessment of each slice includes corresponding T1-weighted spin echo (T1SE) and short tau inversion recovery (STIR) MRI sequences. The T1SE sequence detects the signal from fat and permits detailed assessment of the anatomy of the joint. The STIR sequence is a fat-suppression technique that allows the visualization of the free water signal associated with inflammation and is particularly useful for identifying acute lesions in the bone marrow that may be obscured by marrow fat.

A similar standardized methodology is recommended for assessment of the spine. The Canada-Denmark International MRI Working Group has developed and validated standardized definitions for acute lesions in the spine based on the STIR sequence that incorporate a definition of what constitutes the reference normal STIR signal [12]. These include vertebral corner inflammatory lesions (CILs), which are depicted in reference images (Figure 1) that describe typical lesions as well as set the threshold for detection. Calibration of readers using these tools is essential prior to MRI reading exercises. Imaging of the entire spine for SpA is conducted in the sagittal plane and the spine is visualized in two halves, cervico-thoracic and thoraco-lumbar portions. With this large field of view the cervical vertebrae appear small and CILs may not be readily detected (Figure 1). STIR MRI is subject to physiological motion artifacts so that blood flowing in the inferior vena cava and the abdominal aorta may cause spurious STIR signal, termed phase encoding artifacts, which may resemble anterior CILs in the lumbar spine (Figure 1). Consequently, achieving reliability for detection of CILs is challenging even for experienced readers [13] and MRI studies focused on these lesions should include at least two readers scoring independently, with the primary analysis being focused on concordant data from independent reader assessments. 

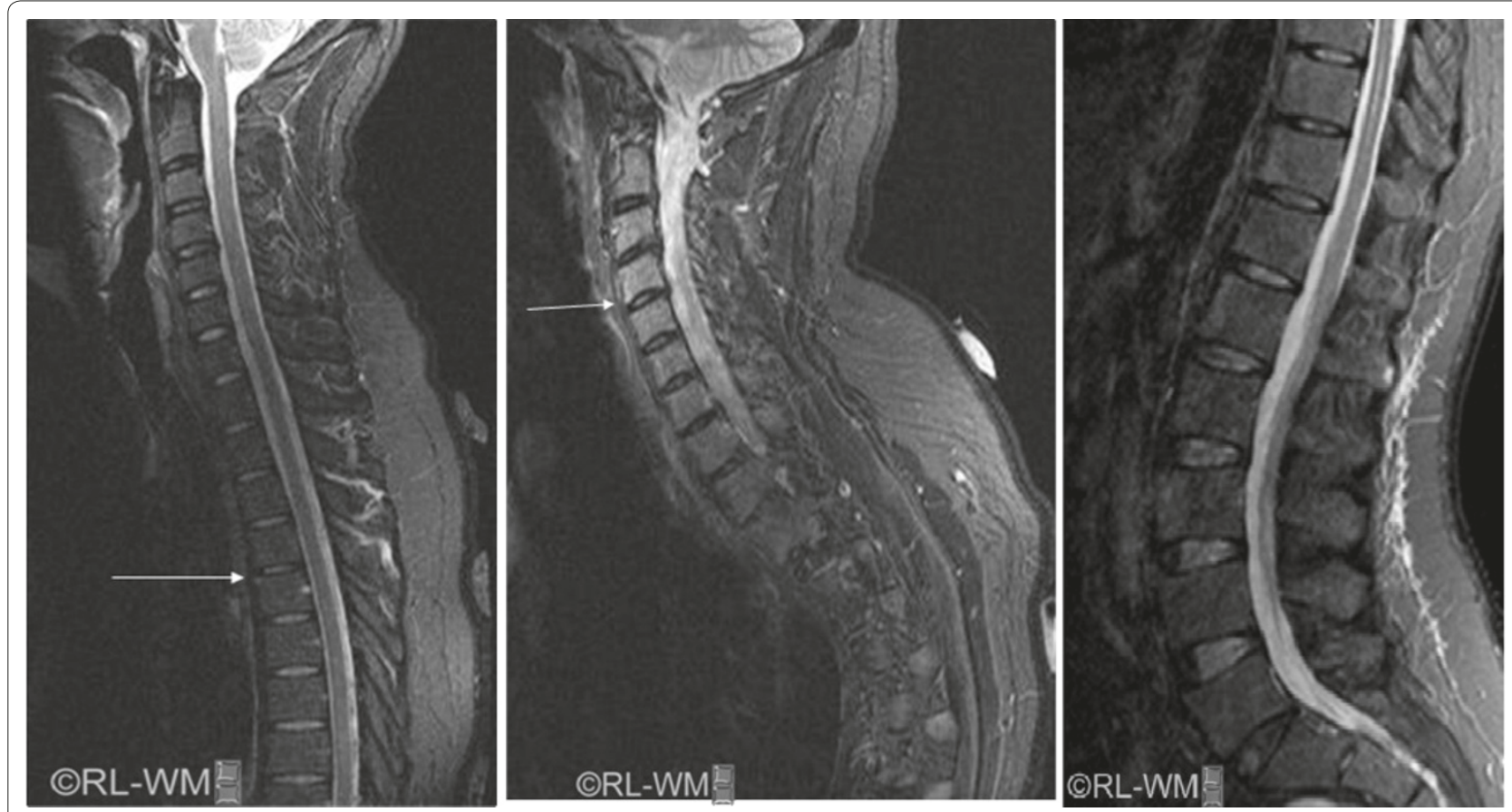

Figure 1. Reference images (Canada-Denmark International MRI Group) of STIR MRI, illustrating the sagittal view of the cervico-thoracic spine. (Reference images are also available at [44].) The arrow in the left image at T5 upper shows a vertebral corner inflammatory lesion (CIL) at the threshold of detection. The bone marrow signal in the center of the vertebral body, if normal, constitutes the reference for designation of normal signal [1 1]. The arrow in the central image shows a CIL at the anterior corner of C4 lower. The large field of view necessary to scan the entire spine in AS impairs detailed assessment of cervical vertebrae. The right image shows increased STIR signal in a vertical pattern across the anterior portions of the lumbar vertebrae simulating inflammation but indicative of blood flowing through the great vessels (phase encoding artifact). STIR, short tau inversion recovery.

It is apparent that the appearance of a CIL may be heterogeneous and the Canada-Denmark group has defined two categories of CIL. In type A CILs the STIR signal is relatively homogeneous and extends to the vertebral corner. In type B CILs, the STIR signal has receded from the vertebral corner and is typically less intense than a type A CIL and more heterogeneous (Figure 2). It is necessary to examine the corresponding T1SE sequence to determine whether there is an erosion or new bone at the vertebral corner, which then appears dark on both the T1SE and STIR sequence, or whether there is tissue metaplasia to fat, which then appears bright on the T1SE sequence. Type B CILs likely represent a more advanced stage of evolution of the inflammatory lesion. In particular, prospective analysis of MRI scans has directly shown that CILs evolve into fat lesions at the corresponding vertebral corner and that this is more likely to occur in patients receiving anti-TNF $\alpha$ therapy $[14,15]$. Reliable detection of type B CILs and assessment of resolution following anti-TNF $\alpha$ therapy are often more difficult than for type A CILs, especially in the lumbar spine due to phase encoding artifacts. Nevertheless, it may be important to make this distinction because the two types of CIL may carry different prognostic implications for the development of new bone (see below).

The approach to the detection of structural lesions by MRI has been the same as for acute lesions. In particular, the proposed standardized definitions for fat infiltration and erosions in both the SIJs and spine have now been validated and reference images are available to set the threshold for detection, to facilitate widespread implementation, and to minimize discrepancies between studies $[16,17]$. The MRI exercises undertaken by the CanadaDenmark and MORPHO study groups have highlighted several key challenges in the reliable assessment of structural lesions. Detection of erosions in the SIJ is particularly challenging without extensive calibration of readers [18]. In particular, while several reports have defined bone erosion as full thickness loss of the dark appearance of either iliac or sacral cortical bone of the SIJ with loss of the adjacent marrow signal on T1-Weighted images, this is often difficult to distinguish from bone sclerosis [19]. Detection of discovertebral erosions can be reliably accomplished with minimal reader calibration but this is more difficult for vertebral corner erosions because these lesions are small and it is difficult to be certain that there is a breach of cortical bone. While the 


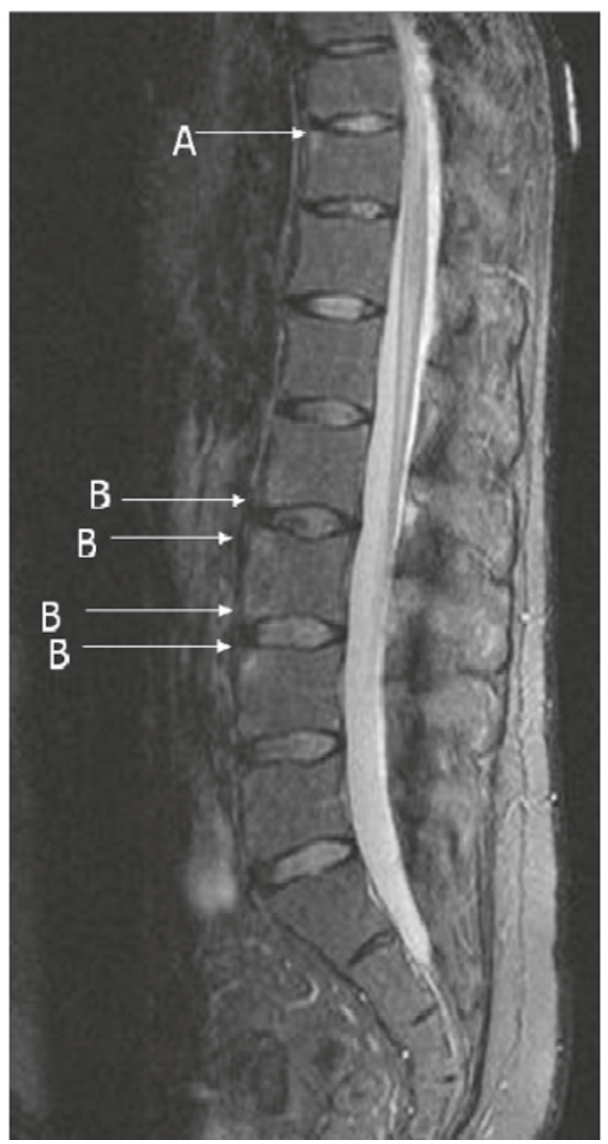

Figure 2. Reference image of short tau inversion recovery MRI illustrating type $\mathrm{A}$ and type $\mathrm{B}$ corner inflammatory lesions. In the latter, the increased short tau inversion recovery MRI signal has receded from the vertebral corner.

detection of fat infiltration is reliably accomplished with minimal calibration, fat infiltration is observed in the SIJs and spine of normal individuals and there is considerable heterogeneity in its morphological characteristics, particularly in the SIJs. It is presently unclear which features relate specifically to $\mathrm{SpA}$.

\section{Prospective studies of MRI inflammation and radiographic progression?}

\section{Spine}

Several studies, all using different methodological approaches, have examined the relationship between inflammation visible on baseline STIR MRI of the spine in patients with established AS and the development of syndesmophytes on radiographs after 2 years of follow up [20-26]. It is important to note from the outset that all radiographic assessments in each study were confined to the anterior cervical and lumbar spine vertebral corners because thoracic spine abnormalities cannot be reliably detected on radiography due to overlapping structures. Similarly, assessment of posterior vertebral corner abnormalities is unreliable [27]. Consequently, MRI assessments have been confined to the corresponding anterior vertebral corners of the cervical and lumbar spine. As discussed above, this restricts MRI assessment to regions of the spine that may pose challenges to the reliable detection of lesions on the STIR sequence. A summary of the findings from these reports is presented in Table 1 and shows that the odds ratios (ORs) for the association of baseline inflammation and the development of new syndesmophytes after 2 years were significant for each study but varied from 1.9 to 4.6 . The percentage of individual vertebral units with inflammation on baseline STIR MRI also varied from 7.5 to $17.2 \%$, the percentage of vertebral regions with baseline inflammation that developed new syndesmophytes varied from 6.5 to $20 \%$, and the percentage of syndesmophytes developing in the absence of baseline inflammation varied from 61 to $76 \%$. So while there is consistency in demonstrating a link between inflammation and new syndesmophytes, it has also been argued that these data more strongly support a lack of coupling between inflammation and new bone because most new syndesmophytes developed from vertebral corners that appeared normal on STIR MRI. What could account for the differences between studies and how does this shed light on the hypothesis that inflammation and ankylosis are coupled and/or uncoupled?

The first report assessed inflammation on baseline STIR MRI in 39 patients who participated in clinical trials of anti-TNF $\alpha$ therapy [20]. Inflammation was recorded at vertebral edges (VEs), and these were defined as positive for inflammation 'if the inflammatory activity was present in the anterior half of the vertebral edge only' This could refer to lesions directly at the vertebral corners as well as lesions adjacent to the endplate but not extending to the vertebral corner as in a discovertebral lesion, which could have different prognostic implications. The report does not indicate how many readers assessed either the MRI scans or the radiographs. Inflammation was reported in $17.2 \%$ of vertebral edges at baseline, new syndesmophytes developed from $6.5 \%$ of vertebral edges with inflammation as opposed to $2.1 \%$ without inflammation $(\mathrm{OR}=3.3,95 \%$ confidence interval $(\mathrm{CI}) 1.5$ to 7.4 ), and $38 \%$ of all new syndesmophytes developed from vertebral edges with baseline inflammation while $62 \%$ had no baseline inflammation. On follow up at 2 years, $4.4 \%$ of vertebral edges where inflammation had resolved and $5.6 \%$ of vertebral edges that had either persistent inflammation or had newly developed inflammatory lesions developed a new syndesmophyte as compared to $1.3 \%$ of VEs that had no inflammation at either baseline or follow up ( $P=0.07$ and 0.04 , respectively).

The second report assessed inflammation on STIR MRI in 29 patients in anti-TNF $\alpha$ clinical trials and 41 patients 
Table 1. Summary of studies assessing the relationship between inflammation on baseline STIR MRI of the spine and development of new syndesmophytes after 2 years of follow up

\begin{tabular}{|c|c|c|c|c|}
\hline \multirow[b]{2}{*}{ Study } & \multicolumn{2}{|c|}{$\begin{array}{l}\text { Number of patients with new } \\
\text { syndesmophytes at } 2 \text { years }\end{array}$} & \multirow[b]{2}{*}{ OR $(95 \% \mathrm{Cl})$} & \multirow[b]{2}{*}{$P$-value } \\
\hline & Yes & No & & \\
\hline \multicolumn{5}{|l|}{ Baraliakos et al. [19] } \\
\hline Baseline VE+ $(n=163 ; 17.2 \%)$ & $10(6.5 \%)$ & $153(93.5 \%)$ & $3.1(1.4-7.1)$ & 0.007 \\
\hline Baseline VE- $(n=785 ; 82.8 \%)$ & $16(2.1 \%)$ & $769(97.9 \%)$ & & \\
\hline \multicolumn{5}{|l|}{ Maksymowych et al. [20] } \\
\hline \multicolumn{5}{|l|}{ Anti-TNFa trial patients } \\
\hline Baseline CIL+ $(n=30)$ & $6(20 \%)$ & $24(80 \%)$ & $4.6(1.7-12.6)$ & 0.007 \\
\hline Baseline CIL- $(n=370)$ & $19(5.1 \%)$ & $351(94.9 \%)$ & & \\
\hline \multicolumn{5}{|l|}{ Observational cohort patients } \\
\hline Baseline CIL+ $(n=28)$ & $6(15.8 \%)$ & $32(84.2 \%)$ & $6.3(2.4-16.7)$ & 0.001 \\
\hline Baseline CIL- $(n=617)$ & $23(2.9 \%)$ & 778 (97.1\%) & & \\
\hline \multicolumn{5}{|l|}{ Van der Heijde et al. [21] } \\
\hline Baseline DVU+ $(n=297)$ & $36(12.1 \%)$ & $261(87.9 \%)$ & $1.9(1.3-2.8)$ & 0.002 \\
\hline Baseline DVU- $(n=1,707)$ & $115(6.7 \%)$ & $1,592(93.3 \%)$ & & \\
\hline
\end{tabular}

CIL, vertebral corner inflammatory lesion [20]; DVU, discovertebral unit [21]; OR, odds ratio; STIR, short tau inversion recovery; VE, vertebral edge [19].

followed in an observational cohort (23 on standard therapies, 18 receiving anti-TNF $\alpha$ therapy) [21]. Vertebral CILs were assessed as defined by the Canada-Denmark group [12] and the primary analysis focused on concordant data from three reader pairs, each reader scoring MRI scans independently. For the primary reader pair and scans from patients in anti-TNF $\alpha$ trials, inflammation was reported in $7.5 \%$ of vertebral corners at baseline, new syndesmophytes developed from $20 \%$ of CILs at the corresponding vertebral corner as opposed to $5.1 \%$ without CILs (OR $=4.6,95 \%$ CI 1.7 to 12.6 ), and $24 \%$ of all new syndesmophytes developed from CILs while $76 \%$ had no baseline inflammation. Data were comparable for scans from patients in the observational cohort (Table 1). On follow up at 2 years, $25 \%$ of CILs where inflammation had resolved had developed a new syndesmophyte compared to none of the CILs that had persistent inflammation. The follow-up data on the observational cohort were extended to include 23 patients receiving anti-TNF $\alpha$ therapy and 27 receiving standard therapy and reported in a separate manuscript [24]. Consistent with the earlier report, new syndesmophytes developed from CILs that resolved by 2 years $(42.9 \%$ in anti-TNF $\alpha$ and $20 \%$ in standard therapy groups, respectively) but not from a single CIL where inflammation was persistent.

A third report assessed the relationship between inflammation on baseline STIR MRI and development of new syndesmophytes but did not directly assess inflammation at the corresponding vertebral corner [22]. Inflammation was recorded in MRI scans from 182 patients in a trial of infliximab according to its presence/ absence at each cervical and lumbar discovertebral unit (DVU), which represents the region between two imaginary lines drawn through the middle of two adjacent vertebrae and includes the four vertebral corners, the intervertebral disc, the adjacent vertebral endplates and bone marrow [28]. Data for each of two readers scoring independently were reported but not concordant data. Inflammation was reported in $14.8 \%$ and $20 \%$ of DVUs at baseline for readers 1 and 2, respectively. For the primary reader, new syndesmophytes developed from $12.1 \%$ of DVUs with inflammation as opposed to $6.7 \%$ without inflammation (OR $=1.9,95 \%$ CI 1.3 to 2.8 ), and $23.8 \%$ of all new syndesmophytes developed from DVUs with baseline inflammation while $76.2 \%$ had no baseline inflammation. At the patient level, MRI activity was not associated with change in radiography score.

The studies that assessed the association between baseline inflammation and new syndesmophytes at the corresponding vertebral corner $[20,21]$ reported higher ORs than the study that assessed inflammation at the level of a DVU [22]. This, as well as the absence of an association between MRI activity and change in radiography score at the patient level [22], is not surprising. As shown in Figure 3, an inflammatory lesion can be observed in several locations within a DVU other than the vertebral corner where a new syndesmophyte develops and this will serve to reduce the strength of the association.

The consensus that most new syndesmophytes developed from vertebral corners that did not demonstrate inflammation at baseline should not necessarily be 


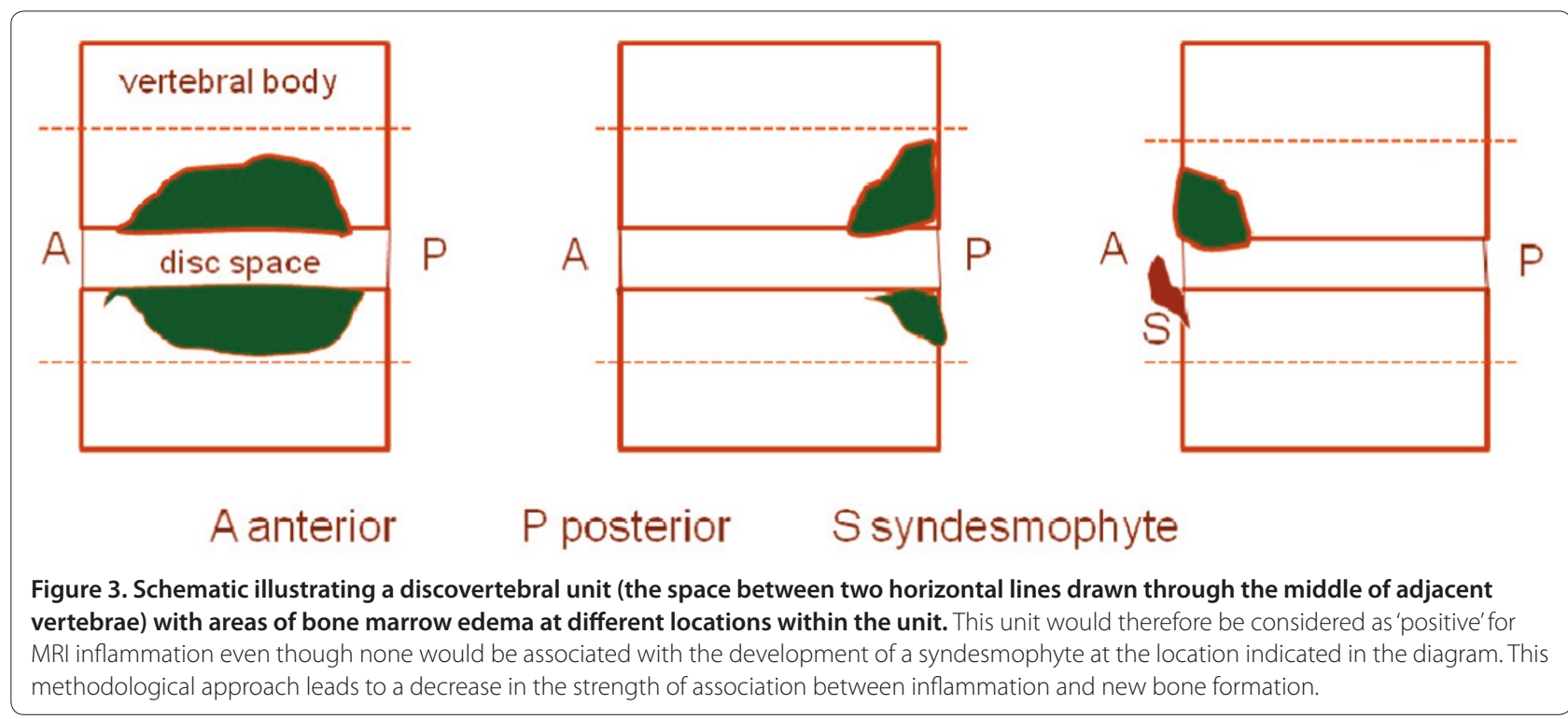

interpreted as demonstrating uncoupling between inflammation and radiographic progression. First, MRI detects only about half of the inflammatory lesions evident histopathologically in the spine of patients with AS [29]. Second, the baseline MRI represents a snapshot in time and new inflammatory lesions can develop even in patients on anti-TNF $\alpha$ agents [20]. Third, prospective studies show that inflammatory lesions evolve into fat lesions evident on T1SE MRI and this can be seen by 52 weeks after the start of treatment, especially in patients on anti-TNF $\alpha$ agents [14,15]. This is relevant because two recent reports have demonstrated an association between baseline fat lesions at vertebral corners on T1SE MRI and the development of new syndesmophytes at the corresponding corner [23,26]. The first assessed 100 MRI scans from 2 cohorts of patients with AS, a clinical trial cohort of patients receiving antiTNF $\alpha$ therapy $(n=38)$ and an observational cohort ( $\mathrm{n}=62,26$ receiving anti-TNF $\alpha$ and 36 receiving standard therapies), while the second report assessed 76 scans from a trial of adalimumab. Consequently, since fat lesions represent a more advanced stage in the evolution of an inflammatory lesion and are themselves associated with radiographic progression, further studies should assess both STIR and T1SE MRI scans before concluding that a syndesmophyte has developed from a vertebral corner without current or prior inflammation.

Another report analyzed the association between change in MRI SIJ inflammation score after 22 weeks of anti-TNF $\alpha$ therapy and the development of new syndesmophytes [25]. Patients developing new syndesmophytes had larger reductions in MRI SIJ inflammation score and decreases in C-reactive protein (CRP) and IL6 so that normalization of CRP and IL6 was more frequently observed in patients with new syndesmophytes even after adjustment for the extent of radiographic damage at baseline. These patient level data demonstrating an association between resolution of inflammation and new bone formation are consistent with the data reported at the individual vertebral corner level [21,24]. It is important to note, however, that the predictive capacity of biomarkers associated with inflammation for new bone is presently unclear [30,31]. CRP has been inconsistently associated with development of new bone $[32,33]$, while IL6 has not been shown to be a predictor, which may reflect its complex role in inflammation and bone metabolism [34,35].

A major consideration in the analysis of the association between MRI inflammatory and other lesions such as fat and the development of new bone is the necessity to adjust for within-patient variation in the extent of radiographic damage at baseline because it has been shown previously that the likelihood of finding a new syndesmophyte is higher in a patient that already has syndesmophytes and/ or ankylosis at baseline [36]. One study reported that the association between inflammation at the level of a DVU and new bone remained statistically significant after adjustment for such within-patient correlation as well as after further adjustment for other confounders [22]. A second report described the use of generalized linear latent and mixed models (GLLAMMs) to show that vertebral corners that were inflammation-positive significantly predicted new syndesmophytes after adjustment for within-patient dependence in the total number of vertebral corners with fat, inflammation, and syndesmophytes/ankylosis at baseline [23].

It may be possible that very early inflammatory type A CILs resolve completely without any sequelae if anti-TNF 
therapy is introduced before bone formation pathways become activated [37]. On the other hand, once a lesion has become more advanced as in a type B CIL and crossed a certain 'threshold' of maturation, introduction of anti-TNF therapy may alleviate inflammation but bone formation may even be enhanced through downregulation of Dickkopf-1, a major inhibitor of bone formation by inhibiting signaling through Wingless proteins [38]. TNFo is a major positive regulator of Dickkopf-1. For an individual patient the overall development of new bone during anti-TNF therapy may depend, therefore, on the balance between the number of early and more mature inflammatory lesions. This hypothesis could explain the lack of impact of anti-TNFo therapies on radiographic progression. A recent report supports this hypothesis, which will require further testing in early SpA cohorts [26].

\section{Sacroiliac joint}

Several small studies have assessed inflammatory changes in the SIJ as detected by increased STIR signal reflecting bone marrow edema in subchondral bone and the subsequent development of radiographic sacroiliitis. One study evaluated 17 patients with inflammatory back pain of 3 to 14 months duration but normal pelvic X-ray who were followed for 18 to 30 months [39]. Virtually all patients had abnormalities on baseline MRI that persisted on follow-up MRI 2 to 30 months later and 11 developed plain radiographic features of sacroiliitis on follow up, suggesting that inflammation observed on MRI is of prognostic significance. In a second prospective study of 25 consecutive HLA-B27-positive patients with inflammatory low back pain and $\leq$ grade 2 unilateral sacroiliitis, the positive predictive value of $\geq$ grade 2 sacroiliitis on baseline MRI for the development of $\geq$ grade 2 sacroiliitis on plain radiograph after 3 years was $60 \%$ (sensitivity $85 \%$, specificity $47 \%$ ) [40]. In contrast to the previous study, subchondral marrow edema was found in only 20 SIJs and median duration of symptoms was much longer (4 years), raising questions as to the reliability of the clinical diagnosis. In a study of 40 consecutive patients presenting with inflammatory back pain according to the Calin criteria and followed for a mean of 7.7 years [41], the combination of severe bone marrow edema with HLA-B27 positivity was a predictor of radiographic sacroiliitis (likelihood ratio 8.0, specificity 92\%) while minor bone marrow edema, which can be found in about $25 \%$ of healthy age and sex-matched healthy controls [10], had no predictive validity. However, a detailed quantitative analysis of bone marrow edema in this cohort has recently reported that about a third of patients with substantial bone marrow edema still had minimal radiographic changes after 7.7 years of follow up [42]. Although this implies additional unidentified prognostic factors, follow-up MRI examination was not available so it is unclear to what extent these inflammatory features were persistent.

\section{Future directions}

It is likely that attention will now focus on patients with early disease to further understand the prognostic capacity of MRI. In particular, the development of the new Assessment of SpondyloArthritis international Society (ASAS) classification criteria has broadened the spectrum of disease to include patients with nonradiographic disease [43]. While MRI is now accepted as a classification criterion, its prognostic role is still uncertain. Preliminary evidence suggests that bone marrow edema alone may have insufficient prognostic capacity. Increasing attention is therefore also turning towards other features on MRI, such as fatty lesions and erosions. Although these are often termed 'chronic' lesions, they may be seen in a substantial proportion of patients with non-radiographic SpA [10] but little is known regarding their prognostic capacity. There is keen interest in the assessment of spinal inflammatory lesions in early disease and comprehensive follow up of sufficient duration that will allow greater understanding of the associations between acute and structural lesions as well as between different structural lesions. These longitudinal data will be an essential prerequisite to the development of imaging surrogates that may supplant radiographic progression and facilitate the development of novel disease-modifying therapies.

This article is part of the series Advances in the imaging of rheumatic diseases, edited by Mikkel Østergaard. Other articles in this series can be found at http://arthritis-research.com/series/imaging

\section{Abbreviations}

AS, ankylosing spondylitis; Cl, confidence interval; ClL, corner inflammatory lesion; CRP, C-reactive protein; DVU, discovertebral unit; IL, interleukin; MRI, magnetic resonance imaging; OR, odds ratio; SIJ, sacroiliac joint; SpA, spondyloarthritis; STIR, short tau inversion recovery; T1SE, T1-weighted spin echo; $T N F$, tumor necrosis factor; $V E$, vertebral edge.

\section{Competing interests}

The author has received honoraria and/or unrestricted grants from Pfizer, Merck, Abbott, Amgen, Eli-Lilly, Janssen.

\section{Acknowledgements}

Walter P Maksymowych is a Scientist of the Alberta Heritage Foundation for Medical Research.

\section{Published: 19 April 2012}

\section{References}

1. Ball J: Enthesopathy of rheumatoid and ankylosing spondylitis. Ann Rheum Dis 1971, 30:213-223.

2. van der Heijde D, Landewé R, Einstein S, Ory P, Vosse D, Ni L, Lin SL, Tsuji W, Davis JC Jr: Radiographic progression of ankylosing spondylitis after up to two years of treatment with etanercept. Arthritis Rheum 2008, 58:1324-1331

3. van der Heijde D, Landewé R, Baraliakos X, Houben $H$, van Tubergen A, 
Williamson P, Xu W, Baker D, Goldstein N, Braun J; Ankylosing Spondylitis Study for the Evaluation of Recombinant Infliximab Therapy Study Group: Radiographic findings following two years of infliximab therapy in patients with ankylosing spondylitis. Arthritis Rheum 2008, 58:3063-3070.

4. van der Heijde D, Salonen D, Weissman BN, Landewé R, Maksymowych WP, Kupper H, Ballal S, Gibson E, Wong R; Canadian (M03-606) study group; ATLAS study group: Assessment of radiographic progression in the spines of patients with ankylosing spondylitis treated with adalimumab for up to 2 years. Arthritis Res Ther 2009, 11:R127.

5. Wanders A, Heijde D, Landewé R, Béhier JM, Calin A, Olivieri I, Zeidler H, Dougados M: Nonsteroidal anti-inflammatory drugs reduce radiographic progression in patients with ankylosing spondylitis: a randomized clinical trial. Arthritis Rheum 2005, 52:1756-1765.

6. Lories RJ, Derese I, de Bari C, Luyten FP: Evidence for uncoupling of inflammation and joint remodeling in a mouse model of spondylarthritis. Arthritis Rheum 2007, 56:489-497.

7. Lories RJ, Luyten FP, de Vlam K: Progress in spondylarthritis. Mechanisms of new bone formation in spondyloarthritis. Arthritis Res Ther 2009, 11:221.

8. Sieper J, Appel H, Braun J, Rudwaleit M: Critical appraisal of assessment of structural damage in ankylosing spondylitis. Arthritis Rheum 2008, 58:649-656

9. SPARCC MRI [http://www.arthritisimaging.ca/]

10. Weber U, Lambert RG, Ostergaard M, Hodler J, Pedersen SJ, Maksymowych WP: The diagnostic utility of MRI in spondyloarthritis: An international multicentre evaluation of 187 subjects (The MORPHO study). Arthritis Rheum 2010, 62:3048-3058.

11. Maksymowych WP, Dhillon SS, Chiowchanwisawakit P, Pedersen SJ, Martinez B, Østergaard M, Lambert RGW: Development and validation of web-based training modules for systematic evaluation of active inflammatory lesions in the spine and sacroiliac joints in spondyloarthritis. J Rheumatol 2009, 36(suppl 84):48-57.

12. Lambert RGW, Pedersen SJ, Maksymowych WP, Chiowchanwisawakit P, Østergaard M: Active inflammatory lesions detected by magnetic resonance imaging in the spine of patients with spondyloarthritis definitions, assessment system, and reference image set. J Rheumatol 2009, 36(suppl 84):3-17.

13. Pedersen SJ, Østergaard M, Chiowchanwisawakit P, Lambert RGW, Maksymowych WP: Validation of definitions for active inflammatory lesions detected by magnetic resonance imaging in the spine of patients with spondyloarthritis. J Rheumatol 2009, 36(suppl 84):35-38.

14. Chiowchanwisawakit $P$, Lambert RG, Maksymowych WP: What is the association between inflammation and focal fat infiltration in AS and does treatment matter [abstract]? Ann Rheum Dis 2010, 69(suppl3):262.

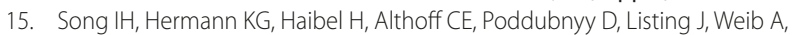
Freundlich B, Rudwaleit M, Sieper J: Relationship between active infl ammatory lesions in the spine and sacroiliac joints and new development of chronic lesions on whole-body MRI in early axial spondyloarthritis: results of the ESTHER trial at week 48. Ann Rheum Dis 2011, 70:1257-1263.

16. Østergaard M, Maksymowych WP, Pedersen SJ, Chiowchanwisawakit P, Lambert RGW: Structural lesions detected by magnetic resonance imaging in the spine of patients with spondyloarthritis - definitions, assessment system, and reference image set. J Rheumatol 2009, 36(suppl 84):18-34.

17. Chiowchanwisawakit P, Østergaard M, Pedersen SJ, Lambert RGW, ConnerSpady B, Maksymowych WP: Validation of definitions for structural lesions detected by magnetic resonance imaging in the spine of patients with spondyloarthritis. J Rheumatol 2009, 36(suppl 84):39-47.

18. Weber U, Pedersen SJ, Ostergaard M, Rufibach K, Lambert RG, Maksymowych WP: Erosions on MRI of the sacroiliac joints in patients with ankylosing spondylitis: can they be reliably detected [abstract]? Ann Rheum Dis 2011 70 (Suppl3):83.

19. Rudwaleit M, Jurik AG, Hermann KG, Landewé R, van der Heijde D, Baraliakos X, Marzo-Ortega H, Ostergaard M, Braun J, Sieper J: Defining active sacroilitis on magnetic resonance imaging (MRI) for classification of axial spondyloarthritis: a consensual approach by the ASAS/OMERACT MRI group. Ann Rheum Dis 2009, 68:1520-1527.

20. Baraliakos X, Listing J, Rudwaleit M, Sieper J, Braun J: The relationship between inflammation and new bone formation in patients with ankylosing spondylitis. Arthritis Res Ther 2008, 10:R104.

21. Maksymowych WP, Chiowchanwisawakit P, Clare T, Pedersen SJ, Østergaard M, Lambert RG: Inflammatory lesions of the spine on MRI predict the development of new syndesmophytes in ankylosing spondylitis: evidence of a relationship between inflammation and new bone formation. Arthritis Rheum 2009, 60:93-102

22. Van der Heijde D, Landewé R, Baraliakos, Hermann K, Houben H, Hsu B, Baker $\mathrm{D}$, Braun J: MRI-inflammation of the vertebral unit (vu) only marginally contributes to new syndesmophyte formation in that unit: a multi-level analysis. Ann Rheum Dis 2008, 67(Suppl II):130.

23. Chiowchanwisawakit P, Lambert RGW, Conner-Spady B, Maksymowych WP: Focal fat lesions at vertebral corners on magnetic resonance imaging predict the development of new syndesmophytes in ankylosing spondylitis. Arthritis Rheum 2011, 63:2215-2225.

24. Pedersen SJ, Chiowchanwisawakit P, Lambert RG, Østergaard M, Maksymowych WP: Resolution of inflammation following treatment of ankylosing spondylitis is associated with new bone formation. J Rheumatol 2011, 38:1349-1354.

25. Pedersen SJ, Sørensen IJ, Lambert RG, Hermann KG, Garnero P, Johansen JS, Madsen OR, Hansen A, Hansen MS, Thamsborg G, Andersen LS, Majgaard O, Loft AG, Erlendsson J, Asmussen KH, Jurik AG, Møller J, Hasselquist M, Mikkelsen D, Østergaard M: Radiographic progression is associated with resolution of systemic inflammation in patients with axial spondyloarthritis treated with TNFa inhibitors. A study of radiographic progression, MRI inflammation and circulating biomarkers of inflammation, angiogenesis, and cartilage and bone turnover. Arthritis Rheum 2011, 63:3789-3800.

26. Maksymowych WP, Morency N, Lambert RG: Evidence for autonomous new bone formation in advanced inflammatory lesions in the spine of patients with ankylosing spondylitis. Ann Rheum Dis 2011, 70 (Suppl3):97.

27. Wanders AJ, Landewé RB, Spoorenberg A, Dougados M, van der Linden S, Mielants $\mathrm{H}$, van der Tempel $\mathrm{H}$, van der Heijde DM: What is the most appropriate radiologic scoring method for ankylosing spondylitis? A comparison of the available methods based on the Outcome Measures in Rheumatology Clinical Trials filter. Arthritis Rheum 2004, 50:2622-2632.

28. Braun J, Baraliakos X, Golder W, Brandt J, Rudwaleit M, Listing J, Bollow M, Sieper J, Van Der Heijde D: Magnetic resonance imaging examinations of the spine in patients with ankylosing spondylitis, before and after successful therapy with infliximab: evaluation of a new scoring system. Arthritis Rheum 2003, 48:1126-1136.

29. Appel H, Loddenkemper C, Grozdanovic Z, Ebhardt H, Dreimann M, Hempfing A, Stein H, Metz-Stavenhagen P, Rudwaleit M, Sieper J: Correlation of histopathological findings and magnetic resonance imaging in the spine of patients with ankylosing spondylitis. Arthritis Res Ther 2006, 8:R143.

30. Maksymowych WP: Biomarkers in spondyloarthritis. Curr Rheumatol Rep 2010, 12:318-324.

31. Maksymowych WP: Biomarkers in spondyloarthritis: from pathophysiology to disease assessment. Joint Bone Spine 2012, 79:4-6.

32. Van der Heijde DM, Wanders A, Mielants H, Dougados M, Landewe RBM: Prediction of progression of radiographic damage over 4 years in patients with ankylosing spondylitis [abstract]. Ann Rheum Dis 2004, 63:OP0132.

33. Maksymowych WP, Haroon N, Morency N, Cook R, Shen H, Rahman P, Gladman DD, Inman RD: Which clinical variables predict radiographic progression in spondyloarthritis? An analysis of the Spondyloarthritis Research Consortium of Canada (SPARCC) Cohort. Arthritis Rheum 2010, 62(10 suppl):S822.

34. Baraliakos X, Landewe R, van der Heijde D, Listing J Baker D, Hsu B, Braun J: A biomarker response is not associated with long-term radiographic progression in AS patients treated with infliximab: a follow-up evaluation of the ASSERT biomarker study. Ann Rheum Dis 2011, 70 (Suppl3):343.

35. Scheller J, Chalaris A, Schmidt-Arras D, Rose-John S: The pro- and antiinflammatory properties of the cytokine interleukin-6. Biochim Biophys Acta 2011, 1813:878-888.

36. Baraliakos X, Listing J, Rudwaleit M, Haibel H, Brandt J, Sieper J, Braun J: Progression of radiographic damage in patients with ankylosing spondylitis: defining the central role of syndesmophytes. Ann Rheum Dis 2007, 66:910-915.

37. Maksymowych WP: What do biomarkers tell us about the pathogenesis of ankylosing spondylitis? Arthritis Res Ther 2009, 11:101-102.

38. Diarra D, Stolina M, Polzer K, Zwerina J, Ominsky MS, Dwyer D, Korb A, Smolen J, Hoffmann M, Scheinecker C, van der Heide D, Landewe R, Lacey D, Richards WG, Schett G: Dickkopf-1 is a master regulator of joint remodeling. Nat Med 2007, 13:156-163.

39. Oostveen J, Prevo R, den Boer J, van de Laar M: Early detection of sacroiliitis on magnetic resonance imaging and subsequent development of 
sacroilitis on plain radiography. A prospective, longitudinal study. J Rheumatol 1999, 26:1953-1958.

40. Blum U, Buitrago-Tellez C, Mundinger A, Krause T, Laubenberger J, Vaith P, Peter $\mathrm{HH}$, Langer M: Magnetic resonance imaging (MRI) for detection of active sacroiliitis-a prospective study comparing conventional radiography, scintigraphy, and contrast enhanced MRI. J Rheumatol 1996, 23:2107-2115

41. Bennett AN, McGonagle D, O'Connor P, Hensor EM, Sivera F, Coates LC, Emery $\mathrm{P}$, Marzo-Ortega H: Severity of baseline magnetic resonance imagingevident sacroiliitis and HLA-B27 status in early inflammatory back pain predict radiographically evident ankylosing spondylitis at eight years. Arthritis Rheum 2008, 58:3413-3418.

42. Aydin SZ, Maksymowych WP, Bennett AN, McGonagle D, Emery P, MarzoOrtega $\mathrm{H}$ : Validation of the ASAS criteria and definition of a positive MRI of the sacroiliac joint in an inception cohort of axial spondyloarthritis followed up for 8 years. Ann Rheum Dis 2012, 71:56-60.
43. Rudwaleit M, van der Heijde D, Landewe R, Listing J, Akkoc N, Brandt J, Braun J, Chou CT, Collantes-Estevez E, Dougados M, Huang F, Gu J, Khan MA, Kirazli Y, Maksymowych WP, Mielants H, Sorensen IJ, Ozgocmen S, Roussou E, ValleOnate R, Weber U, Wei J, Sieper J: The development of Assessment of SpondyloArthritis international Society (ASAS) classification criteria for axial spondyloarthritis (Part II): Validation and final selection. Ann Rheum Dis 2009, 68:777-783.

44. Arthritis Doctor.ca [http://www.arthritisdoctor.ca/]

doi:10.1186/ar3786

Cite this article as: Maksymowych WP: MRI and X-ray in axial spondyloarthritis: the relationship between inflammatory and structural changes. Arthritis Research \& Therapy 2012, 14:207. 